\title{
First-principles search for efficient activators for $\mathrm{LaI}_{3}$
}

\author{
D. Wu ${ }^{\mathrm{a}}$, M. P. Prange ${ }^{\mathrm{a}}$, F. Gao ${ }^{\mathrm{a}, \mathrm{b}}$, S. Kerisit ${ }^{\mathrm{a}, *}$ \\ ${ }^{a}$ Physical Sciences Division, Pacific Northwest National Laboratory, Richland, Washington 99354, USA \\ ${ }^{b}$ Department of Nuclear Engineering and Radiological Science, University of Michigan, Ann Arbor, Michigan 48109, USA
}

\begin{abstract}
First-principles calculations were performed using density functional theory with Hubbard corrections or hybrid exchangecorrelation functionals, as well as the $G W$ approximation, to predict dopants that could serve as efficient activators for $\mathrm{LaI}_{3}$, a potentially very bright scintillator for which an appropriate activator has not been identified yet. The dopants considered in this work included a series of lanthanide ions ( $\mathrm{Ce}, \mathrm{Pr}, \mathrm{Nd}, \mathrm{Eu}, \mathrm{Gd}$, and $\mathrm{Tb}$ ) and several $\mathrm{n}^{2}$ ions $(\mathrm{Tl}, \mathrm{Pb}, \mathrm{Bi}$, and $\mathrm{Sb})$. Based on both ground-state calculations and constrained DFT calculations to simulate excited states, the trivalent lanthanide dopants were shown not to constitute an improvement over $\mathrm{Ce}^{3+}$, for which experimental data exist, as they showed occupied $4 f$ states below the valence band maximum (VBM) and $5 d$ states above the conduction band maximum (CBM). In contrast, the only divalent lanthanide considered, $\mathrm{Eu}^{2+}$, displayed occupied $4 f$ states within the band gap of the host, but its $5 d$ states were calculated to be above the $\mathrm{CBM}^{2} \mathrm{Eu}^{2+}$ could nonetheless be exploited as an activator by increasing the band gap energy slightly through substitution of iodide ions by bromide ions. Similar results were obtained for $\mathrm{Tl}^{+}$. Finally, $\mathrm{Bi}^{3+}$ and $\mathrm{Sb}^{3+}$ were predicted to be efficient activators in $\mathrm{LaI}_{3}$ by virtue of having unoccupied $p$ states below the $\mathrm{CBM}$, which can serve as electron traps and can combine with holes at the VBM to form localized luminescence centers. Therefore, Bi- and Sb-doped $\mathrm{LaI}_{3}$ should be grown and tested experimentally. Comparison with empirical relationships of the relative positions of the energy levels of rare-earth dopants and implications for efficient doping schemes and scintillation mechanisms in $\mathrm{LaI}_{3}$ are also discussed.
\end{abstract}

Keywords: scintillators, density functional theory, activators, electronic structure, luminescence centers

\section{Introduction}

Inorganic scintillators have found applications in a wide range of fields including nuclear nonproliferation, medical imaging, and high-energy physics. Key performance 5 characteristics of these materials include energy resolution, brightness, and decay times and result from the response of the material to the large excitation density gradients created by particle radiation such as $\gamma$-rays in so-called ionization tracks. Therefore, advances in the fundamental ${ }^{20}$ physics of the complex solid-state phenomena that lead to the emission of scintillation photons are expected to

*Corresponding author: sebastien.kerisit@pnnl.gov Preprint submitted to Journal of Luminescence translate directly to science-based performance improvements [1]. This realization has driven significant and recent efforts in developing a better understanding of the condensed matter physics of these materials $[2,3,4,5,6$, 7]. In particular, ever-improving software and handware eapabilities have open the door for first-principles calculations to take a more prominant role in material engineering for improved scintillation performance. First-principles calculations have proven especially useful to compute intrinsic scintillator properties $[2,8,9,10]$ and to determine potentially efficient dopirgo sehemes $11,12,13]$. Because of the often high thermal quenching rates of intrinsic emission in these materials, dopants are generally 

tractive to both electrons and holes. Consequently, the positions of the energy levels of the dopants calculated 65 from first-principles methods can provide valuable guidance in the search for bright scintillators. Similar apschemes that may lead to improved resolution [14, 15, 16]. In this work, this approach was applied to $\mathrm{LaI}_{3}$ using den- 70 sity functional theory (DFT) with Hubbard corrections or hybrid exchange-correlation functionals, whereby the Hubbard on-site correction and the fraction of exact exchange were calibrated to experimental data on pure and Ce-doped $\mathrm{LaI}_{3}$ and then employed to simulate a range of 75 dopants previously untested.

$\mathrm{LaI}_{3}$ is a promising low-bandgap material that is cur40 rently not utilized due to the lack of an appropriate activator. Indeed, Monte Carlo simulations of electron-hole pair production performed in our group predicted $\mathrm{LaI}_{3}$ to have 80 a very high theoretical maximum light yield (i.e. before any quenching processes occur) of 174,000 photons/MeV; tons $/ \mathrm{MeV}$ ) of the calculated maximum light yield was achieved in the lone experiment of $\mathrm{LaI}_{3}$ doped with $\mathrm{Ce}_{85}$ [17. The low measured light yield of $\mathrm{LaI}_{3}$ was attributed to the proximity of the $\mathrm{Ce}^{3+} 5 d$ level to the host conduction band, which led to extensive non-radiative thermal quenching and loss of scintillation light. As a result, $\mathrm{LaI}_{3}$ remains unused, despite its promising physical characteristics. Therefore, first-principles calculations were conducted in this work to identify dopants with apupon the results obtained with $\mathrm{Ce}$ and maximize the light yield of $\mathrm{LaI}_{3}$. Specifically, candidate dopants were selected from two families of dopants: lanthanide ions $\left(\mathrm{Ce}^{3+}, \mathrm{Pr}^{3+},{ }_{95}\right.$ $\mathrm{Nd}^{3+}, \mathrm{Eu}^{2+} / \mathrm{Eu}^{3+}, \mathrm{Gd}^{3+}$, and $\left.\mathrm{Tb}^{3+}\right)$ and $\mathrm{ns}^{2}$ ions $\left(\mathrm{Tl}^{+}\right.$, $\mathrm{Pb}^{2+}, \mathrm{Bi}^{3+}$, and $\left.\mathrm{Sb}^{3+}\right)$. Lanthanides, and particularly $\mathrm{Ce}$ and $\mathrm{Eu}$, are popular dopants in inorganic scintillators thanks to their allowed $5 d$ to $4 f$ transitions, whereas $n s^{2}$ ions exhibit $\mathrm{n} p$ to $\mathrm{n} s$ transitions. The general approach used in this work follows that employed by Canning and co-workers in their publications on Ce-doped [12] and Eudoped [13] materials, whereby DFT calculations are performed to compute the ground-state electronic structure of the host/dopant combination and constrained DFT is used to evaluate whether the highest occupied state of the dopant in the excited state is comprised within the gap of the host.

Beyond the particular problem of $\mathrm{LaI}_{3}$, there exists a fundamental trade-off in the development of bright scintillator materials. A decrease in the host band gap energy generally leads to an increase in the number of electronhole pairs produced but the host gap has to be sufficiently large to accommodate the energy levels of the dopants in the ground and excited states. In a recent publication, Du [18 hypothesized that an electron trapped at a $n s^{2}$ dopant could associate with a localized hole in the valence band to allow for an efficient $\mathrm{n} p^{*}-\mathrm{V}_{\mathrm{k}}$ transition. This alternative recombination mechanism could be used to exploit low-band gap insulators. The first-principles calculations performed in this work will also serve to evaluate this hypothesis.

\section{Computational Methods}

All the calculations were performed using the ab-initio total-energy and molecular dynamics program VASP (Vienna Ab-initio Simulation Package) developed at the Fakultät für Physik of the Universität Wien [19, 20, 21]. The spinpolarized calculations were carried out with the projector augmented wave (PAW) method [22, 23]. The majority of the calculations made use of the generalized gradient approximation (GGA) and the Perdew-Burke-Ernzerhof (PBE) exchange-correlation functional [24].

The Hubbard on-site correction $(\mathrm{GGA}+U)$ was also applied using the rotationally invariant method of Dudarev [25, 26]. In these calculations, a $U_{\text {eff }}$ value of 10.32 $\mathrm{eV}\left(U_{\text {eff }}=U-J\right.$, where the on-site Coulomb interaction 
$U$ and exchange interaction $J$ were $11 \mathrm{eV}$ and $0.68 \mathrm{eV}$, respectively) was applied to the La $4 f$ states, following the results of Czyżyk and Sawatzky on lanthanum copper oxides [27]. As discussed in Sec. 3.1, this correction is required because of the self-interaction error associated with the localized nature of $4 f$ states, and it allows for the correct ordering of the La $5 d$ and $4 f$ unoccupied states. An additional, single on-site correction was applied to the $4 f$ states of all the lanthanide dopants. The determination of the value of $U_{\text {eff }}$ for this additional correction is described in Sec. 3.2.1 A select number of calculations were also performed with the HSE06 hybrid functional 28]. Starting from the HSE06 hybrid functional, the fraction of exact exchange was modified to reproduce either the experimental band gap of $\mathrm{LaI}_{3}$ (Sec. 3.1) or the position of the occupied $4 f$ state of $\mathrm{Ce}^{3+}$ (Sec. 3.2.1). To provide a reference for the density of states of pure $\mathrm{LaI}_{3}$, a calculation was also carried out with the $G W$ approximation [29, 30] at the ${ }^{140}$ GGA structure.

The PAW pseudopotentials for all the elements involved in the calculations were obtained from the VASP database, as recommended in the VASP manual. The La and I PAW pseudopotentials included $5 s, 5 p, 4 f, 5 d$, and $6 s$ states and $^{145}$ $5 s, 5 p$, and $5 d$ states as valence electrons, respectively. Two series of dopants were investigated. The first series consisted of several trivalent lanthanide ions, namely $\mathrm{Ce}^{3+}$ $\left(4 f^{1}\right), \mathrm{Pr}^{3+}\left(4 f^{2}\right), \mathrm{Nd}^{3+}\left(4 f^{3}\right), \mathrm{Eu}^{3+}\left(4 f^{6}\right), \mathrm{Gd}^{3+}\left(4 f^{7}\right)$, and $\mathrm{Tb}^{3+}\left(4 f^{8}\right)$. These specific ions were selected to cover ${ }^{150}$ a range of possible occupancies of the $4 f$ shell. For reasons discussed in Sec. $3.2 .2 \mathrm{Eu}^{2+}\left(4 f^{7}\right)$ was also considered. All the lanthanide PAW pseudopotentials included $5 s, 5 p, 4 f, 5 d$, and $6 s$ states as valence electrons. The second series consisted of several $\mathrm{n} s^{2}$ ions, namely $\mathrm{Tl}^{+},{ }^{155}$ $\mathrm{Pb}^{2+}, \mathrm{Bi}^{3+}$, and $\mathrm{Sb}^{3+}$. These specific ions were selected to vary the nominal charge on the dopant ion for the $6 \mathrm{n} s^{2}$ series and to compare the $5 \mathrm{n} s^{2}$ and $6 \mathrm{n} s^{2}$ valence shells for the trivalent ions. The PAW pseudopotentials of the $6 s^{2}$ ions included $5 d, 6 s$, and $6 p$ states as valence electrons ${ }^{160}$ whereas the Sb PAW pseudopotential only included $5 s$ and $5 p$ states.

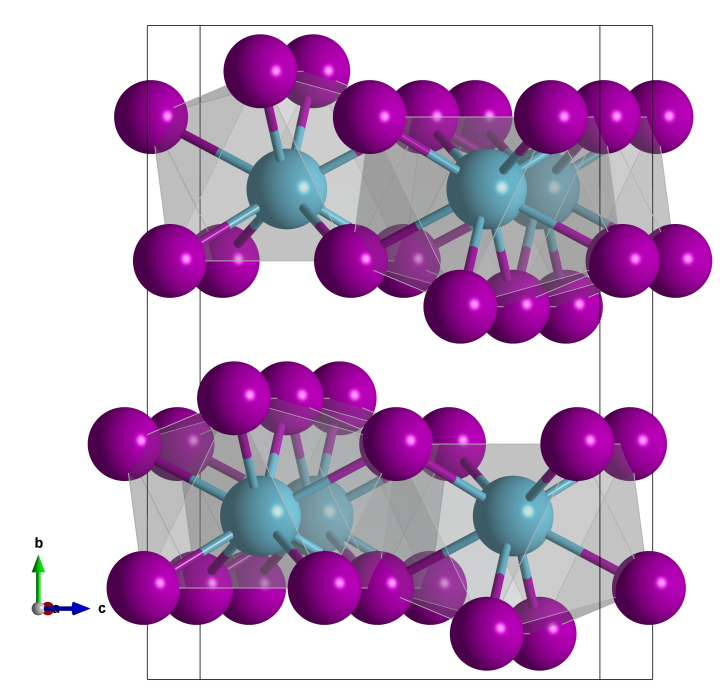

Figure 1: (Color online) Crystal structure of $\mathrm{LaI}_{3}$. The cyan and purple spheres represent La and I atoms, respectively.

Calculations of pure $\mathrm{LaI}_{3}$ were performed with the full unit cell; constant-pressure geometry optimizations (ionic positions, cell volume, and cell shape are allowed to relax) were executed first with GGA and GGA $+U$ with 36 irreducible $k$ points, followed by static calculations with 220 and 16 irreducible $k$ points to compute the band structure with GGA or GGA $+U$ and HSE or $G W$, respectively. Calculations of doped $\mathrm{LaI}_{3}$ made use of a $4 \times 1 \times 2$ supercell constructed from the GGA $+U$ optimized unit cell and containing 128 atoms with one La atom replaced by the dopant element. Constant-volume geometry optimizations (only the ionic positions are allowed to relax) were carried out first with $\mathrm{GGA}+U$ at the $\Gamma$ point, followed by static calculations with 26 and 1 irreducible $k$ points to compute the electronic density of states (DOS) with GGA $+U$ and HSE, respectively. The zero of energy was defined as the top of the valence band in all DOS plots. Convergence was reached when the force exerted on any atom was less than $0.01 \mathrm{eV} / \AA$ in the geometry optimizations and when the total energy change between two electronic steps was less than $10^{-5} \mathrm{eV}$ in the electronic minimizations. The default 
plane-wave cutoff energy set by VASP was used throughout and varied between approximately 250 and $300 \mathrm{eV}$.

Constrained DFT calculations were also performed to simulate excited states. In this approach, the ground-state electronic structure was first obtained at the $\Gamma$ point, then a constrained static calculation was performed, also at the $\Gamma$ point, with fixed occupancies, whereby the highest ground-state occupied $4 f$ state or ns state for the lanthanide or $\mathrm{n} s^{2}$ ions, respectively, was emptied, and one of the lowest unoccupied $5 d$ states for the lanthanide dopants or lowest unoccupied $\mathrm{n} p$ states for the $\mathrm{n} s^{2}$ ions was filled. For the GGA $+U$ calculations, an additional step was performed in which a static calculation was carried out with fixed occupancies and fixed charge density; but with 26 irreducible $k$ points to compute the electronic density of states with the same settings as in the ground-state calculations. Finally, the partial charge density due the promoted band was printed out and the fraction of this density within the Voronoi volume of the dopant $d$, which will be referred to as $\rho_{d}$, was used to quantify the extent of localization of the excited state.

\section{Results}

\subsection{Pure $\mathrm{LaI}_{3}$}

$\mathrm{LaI}_{3}$ crystallizes in an orthorhombic lattice with the
$\mathrm{PuBr}_{3}$ structure (space group $\mathrm{cmcm}$ ). There are 16 atoms in the unit cell, with four La atoms in positions $4(c) \pm\left(0, u, \frac{1}{4} 1 \phi\right.$, four I atoms in positions $4(c) \pm\left(0, v, \frac{1}{4}\right)$, and eight I atoms in positions $8(f) \pm\left(0, m, n ; 0, m, \frac{1}{2}-n\right)$. The lattice constants $(a, b, c)$ obtained from the GGA and GGA $+U$ geometry optimizations are compared to experimental data [31] in Table 1. The structural parameters $(u, v, m, n)$ were215 not provided in that publication. The $a$ and $c$ lattice parameters calculated with GGA are overestimated by $1.7 \%$ and $1.2 \%$, respectively. This is consistent with the fact that GGA generally slightly underestimates binding energies. The $b$ lattice parameters is overestimated by ap-220 proximately $12 \%$. This large error is due to the lack of
Table 1: Comparison of experimental 31 and calculated (GGA and $\mathrm{GGA}+U$ ) lattice parameters of $\mathrm{LaI}_{3}$.

\begin{tabular}{llll}
\hline \hline Parameter & GGA & GGA $+U$ & Exp. [31] \\
\hline$a(\AA)$ & 4.446 & 4.469 & 4.37 \\
$b(\AA)$ & 15.735 & 15.684 & 14.01 \\
$c(\AA)$ & 10.158 & 10.195 & 10.04 \\
$u$ & 0.2591 & 0.2594 & - \\
$v$ & -0.0952 & -0.0925 & - \\
$m$ & 0.3440 & 0.3452 & - \\
$n$ & -0.0670 & -0.0666 & - \\
\hline \hline
\end{tabular}

dispersion interactions, which dominate in this direction due to the close proximity of layers of I atoms, as shown in Fig. 1. Adding a Hubbard correction to the La $4 f$ states only slightly affects the calculated lattice parameters.

The total DOS obtained with GGA is shown in Fig. 2. The resulting band gap energy is $2.1 \mathrm{eV}$. This is lower than the experimental value of $3.3 \mathrm{eV}$ reported by Bessiere et al. [17, which was obtained from the onset of fundamental absorption of Ce-doped $\mathrm{LaI}_{3}$ at $6 \mathrm{~K}$. In addition, based on previous work on lanthanum copper oxides 27 . and $\mathrm{LaBr}_{3}$ [9] as well as our own $G W$ calculations (Fig. 2], the unoccupied La $4 f$ states are incorrectly situated at the bottom of the conduction band, below the La $5 d$ states. The addition of the Hubbard correction described above leads to the correct positioning of the La $5 d$ states at the bottom of the conduction band; however, the band gap energy remains unchanged. Another approach is to employ the HSE hybrid functional and vary the fraction of exact exchange $(\alpha)$ to match the experimental band gap energy. A value of 0.35 was obtained in this way. This procedure also yields the correct ordering of the La $4 f$ and $5 d$ states. Interestingly, the value of $\alpha$ determined here is in very good agreement with those obtained by $\mathrm{Du}[18$ (0.37 to 0.40$)$, when applying the same approach to alkali 
iodides. The partial density of states obtained with the optimized value of $\alpha$ is displayed in Fig. 2 and shows that, as expected based on other iodide materials, the valence ${ }_{240}$

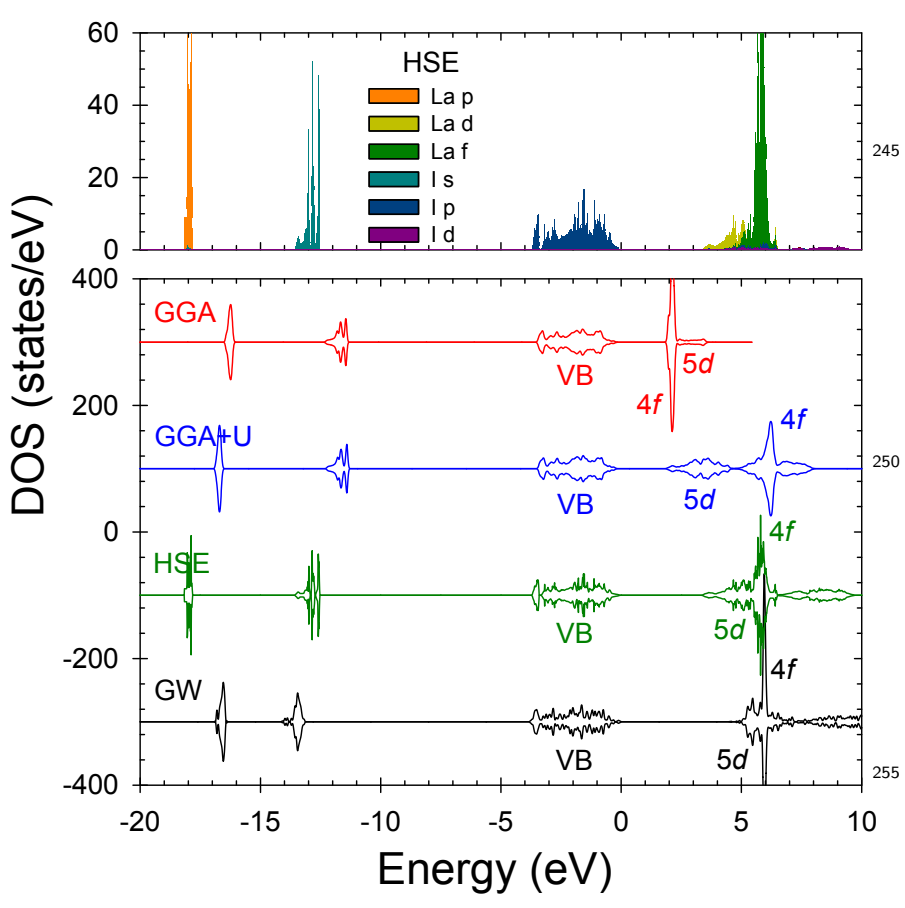

Figure 2: (Color online)(top) Partial DOS of $\mathrm{LaI}_{3}$ from HSE calculation with $\alpha=0.35$ (only up-spin DOS is shown). (bottom) Total DOS from GGA, GGA $+U$, HSE $(\alpha=0.35)$, and $G W$ calculations. Up-spin and down-spin densities of states are shown above and below ${ }^{260}$ the horizontal axis, respectively, in each case.

\subsection{Lanthanide dopants}

\subsubsection{Determination of $U_{\text {eff }}$ for $G G A+U$ calculations and ${ }_{265}$} $\alpha$ for HSE calculations

$\mathrm{Ce}^{3+}$ was first used as a reference because it has been obtained from a 2-state model fit to the temperature dependence of the light yield and decay time $(0.14 \mathrm{eV})$ and (2) the energy difference between the Ce $5 d$ and $4 f$ levels obtained from the sub-bandgap peak in the absorption spectrum $(2.9 \mathrm{eV})$. Therefore, an on-site Hubbard correction was applied to the Ce $4 f$ states and its value was varied until the $4 f$-VBM gap estimated from experiment was reproduced (Fig. 3). A $4 f$-VBM gap of $0.23 \mathrm{eV}$ was obtained for $U_{\text {eff }}=1.7 \mathrm{eV}$. This value is halfway between those reported by Canning et al. [12] (same approach as used here) for Ce-doped $\mathrm{LaI}_{3}$ and Åberg et al. 9] (approach not described) for Ce-doped $\mathrm{LaBr}_{3}$. This optimized value of $U_{\text {eff }}$ will be applied to the $4 f$ states of all the other lanthanide dopants studied in this work.

It should be noted that the value of $3.3 \mathrm{eV}$ corresponds to the optical band gap estimated in [17]; therefore, because of the expected electron-hole interactions, it may correspond to exciton formation rather than the CBM, which would be found slightly higher in energy. Dorenbos [32, 33. reported that the band gap can be estimated to be approximately $8 \%$ greater than the energy of exciton creation, which, following the approach just described, would increase the $4 f$-VBM gap by a few tenths of an eV. As will be evident from the following results and as will be discussed in Sec. 4, this small change in the position of the Ce $4 f$ state would not affect the conclusions of this work.

The optimized structure of Ce-doped $\mathrm{LaI}_{3}$ obtained with $U_{\text {eff }}=1.7 \mathrm{eV}$ was used as the starting point for a series of static calculations performed with HSE at the $\Gamma$ point, in which $\alpha$ was varied to reproduce the $4 f-\mathrm{VBM}$ gap. As $\alpha$ was increased from 0 (equivalent to a PBE calculation), the splitting between the single occupied $4 f$ state and the unoccupied $4 f$ states rapidly increased pushing the occupied $4 f$ state inside the valence band as a result. The fraction of exact exchange that yields the closest agreement with the $4 f$-VBM gap is 0.1 . This value differs from that obtained above to reproduce the experimental band the conduction band minimum $(\mathrm{CBM})$ and the $\mathrm{Ce} 5 d$ level $_{275}$ 
gap energy; therefore, both properties cannot be reproduced with a single value of $\alpha$. Consequently, subsequent calculations of the lanthanide dopants will focus on the GGA $+U$ approach.

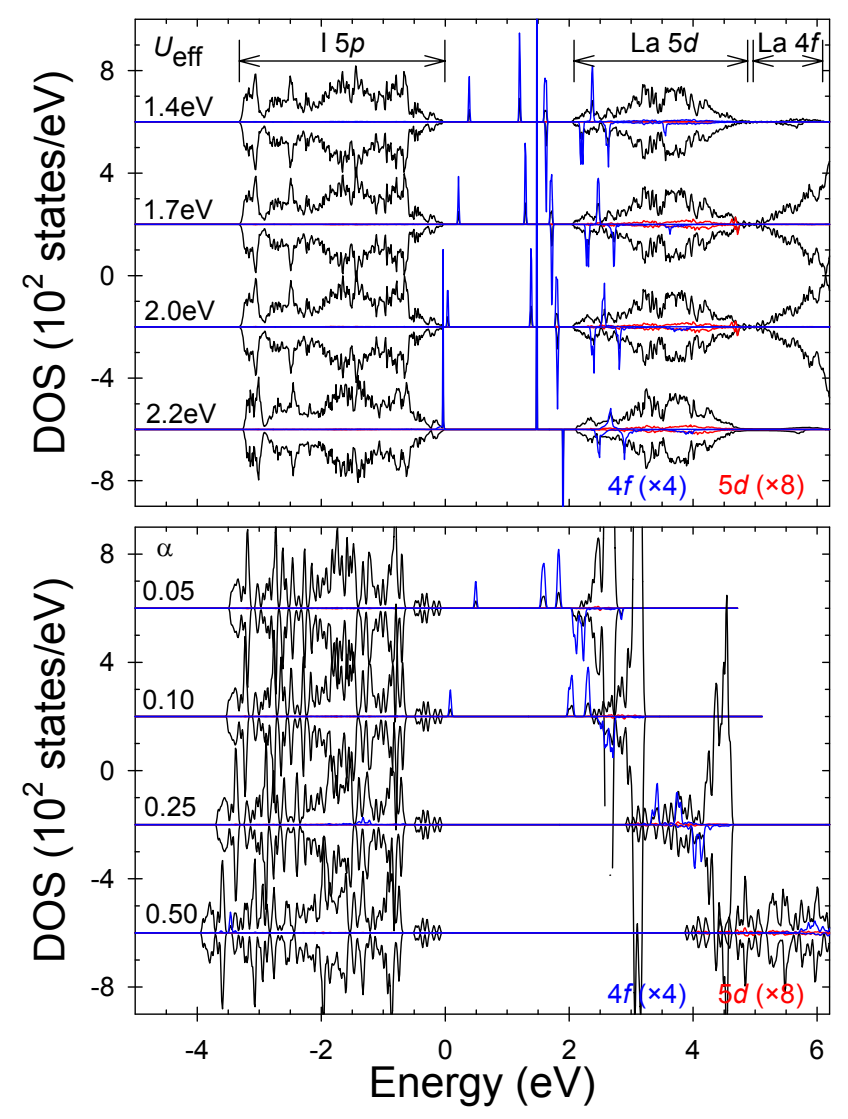

Figure 3: (Color online) Full DOS of Ce-doped $\mathrm{LaI}_{3}$ together with magnified Ce $4 f$ and $5 d$ states, shown in blue and red, respectively, from (top) GGA $+U$ calculations with varying $U_{\text {eff }}$ and (bottom) HSE calculations with varying $\alpha$.

\subsubsection{Ground-state electronic structures}

Constant-volume geometry optimizations were carried out with the $U_{\text {eff }}$ determined for Ce for a $\mathrm{LaI}_{3}$ supercell with a single $\operatorname{Ln}(\mathrm{Ln}=\mathrm{Pr}, \mathrm{Nd}, \mathrm{Eu}, \mathrm{Tb}$, or $\mathrm{Gd})$ atom substituted for La. The Ln dopants were in a high-spin305 configuration in all cases. For Eu, the net spin density was close to that of $\mathrm{Eu}^{2+}$, indicating that $\mathrm{Eu}^{2+}$ is more stable than $\mathrm{Eu}^{3+}$ in $\mathrm{LaI}_{3}$, which is likely due to the additional stability afforded by the half-filled $4 f$ shell for $\mathrm{Eu}^{2+}$. As a result, significant electronic density was drawn from ${ }^{310}$
Table 2: Coordination numbers $(\mathrm{CN})$ and averages $(r)$ and standard deviations $(\sigma)$ of the Ln-I nearest-neighbor distances in calculations of doped $\mathrm{LaI}_{3}$ and in corresponding lanthanide iodide compounds from experimental crystal structures 31, 34, 35, 36.

\begin{tabular}{lcccccc}
\hline \hline & \multicolumn{3}{c}{${\text { in } \mathrm{LaI}_{3} \text { (calc.) }}$} & \multicolumn{3}{c}{ in $\mathrm{Ln}^{+\mathrm{x}} \mathrm{I}_{\mathrm{x}}$ (exp.) } \\
\hline $\mathrm{M}$ & $\mathrm{CN}$ & $r(\AA)$ & $\sigma(\AA)$ & $\mathrm{CN}$ & $r(\AA)$ & $\sigma(\AA)$ \\
\hline $\mathrm{La}$ & 8 & 3.35 & 0.11 & 8 & 3.29 & 0.11 \\
$\mathrm{Ce}$ & 8 & 3.32 & 0.12 & 8 & 3.28 & 0.11 \\
$\mathrm{Pr}$ & 8 & 3.32 & 0.12 & 8 & 3.27 & 0.11 \\
$\mathrm{Nd}$ & 8 & 3.31 & 0.13 & 8 & 3.26 & 0.12 \\
$\mathrm{Eu}$ & 8 & 3.41 & 0.11 & 7 & 3.34 & 0.06 \\
$\mathrm{Gd}$ & 8 & 3.27 & 0.15 & 6 & 3.10 & 0.04 \\
$\mathrm{~Tb}$ & 8 & 3.27 & 0.16 & 6 & 3.09 & 0.04 \\
\hline \hline
\end{tabular}

290 from Levy et al. 34] for the trivalent lanthanide iodides that adopt the $\mathrm{PuBr}_{3}$ structure (La, Ce, Pr, and Nd) and from Trotter and Zobel 35] for those that adopt the $\mathrm{BiI}_{3}$ structure ( $\mathrm{Gd}$ and $\mathrm{Tb})$. As such, these distances represent the best estimates given the available data. For $\mathrm{EuI}_{2}$, however, experimental distances were available from the monoclinic structure resolved by Bärnighausen and Schultz [36]. As expected from the crystal structure of their iodides, $\mathrm{Ce}, \mathrm{Pr}$, and $\mathrm{Nd}$ are very similar to La in terms of size 
and extent of distortion of the first coordination shell. In contrast, Eu, Gd, and $\mathrm{Tb}$ are in a much more constrained coordination environment, although more so for $\mathrm{Gd}$ and ${ }^{335}$ Tb than for Eu. Differences in local coordination environments are likely to influence the ability of the dopants to readily incorporate in the crystal structure of the host.

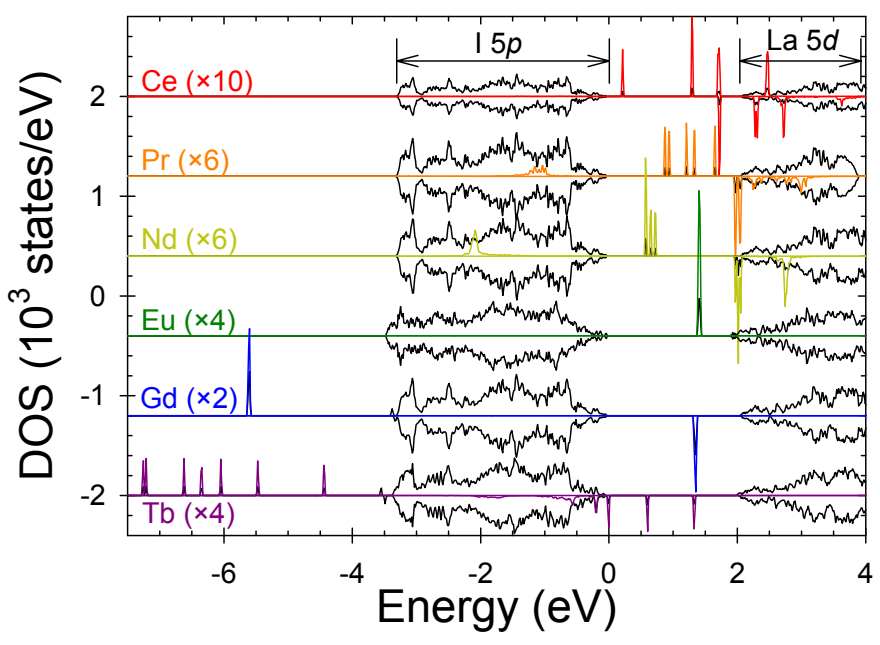

Figure 4: (Color online) Full DOS of $\mathrm{LaI}_{3}$ doped with $\mathrm{Ce}^{3+}, \mathrm{Pr}^{3+}$, $\mathrm{Nd}^{3+}, \mathrm{Eu}^{2+}, \mathrm{Gd}^{3+}$, and $\mathrm{Tb}^{3+}$ obtained from GGA $+U$ calculations. Magnified $4 f$ states of the lanthanide dopants are shown in color.

Fig. 4 shows the Ln $4 f$ states against the full DOS obtained from the GGA+ $U$ calculations. For the trivalent ${ }_{340}$ lanthanides, as the number of $4 f$ electrons increases the energies of the $4 f$ states decrease progressively. As a result, the $4 f$ states of $\operatorname{Pr}^{3+}$ and $\mathrm{Nd}^{3+}$ are within the valence band and those of $\mathrm{Gd}^{3+}$ are several $\mathrm{eV}$ below the bottom of the valence band. Beyond $\mathrm{Gd}^{3+}$, the $4 f$ shell is half full and ${ }_{345}$ additional electrons are in spin down states. For $\mathrm{Tb}^{3+}$, all spin up states are below the valence band and the spin down electron is found towards the VBM. These results indicate that, out of the trivalent lanthanides, only $\mathrm{Ce}^{3+}$ is predicted to be attractive to holes in the valence band by 350 virtue of having an occupied $4 f$ state above the VBM. For the divalent lanthanide $\mathrm{Eu}^{2+}$, the energy of the $4 f$ states is shifted up significantly and is thus found well within the band gap of the host. $\mathrm{Eu}^{2+}$ is therefore also predicted to be attractive to valence band holes. Consequently, onlyзs5
$\mathrm{Ce}^{3+}$ and $\mathrm{Eu}^{2+}$ will be considered further.

\subsubsection{Excited-state electronic structures}

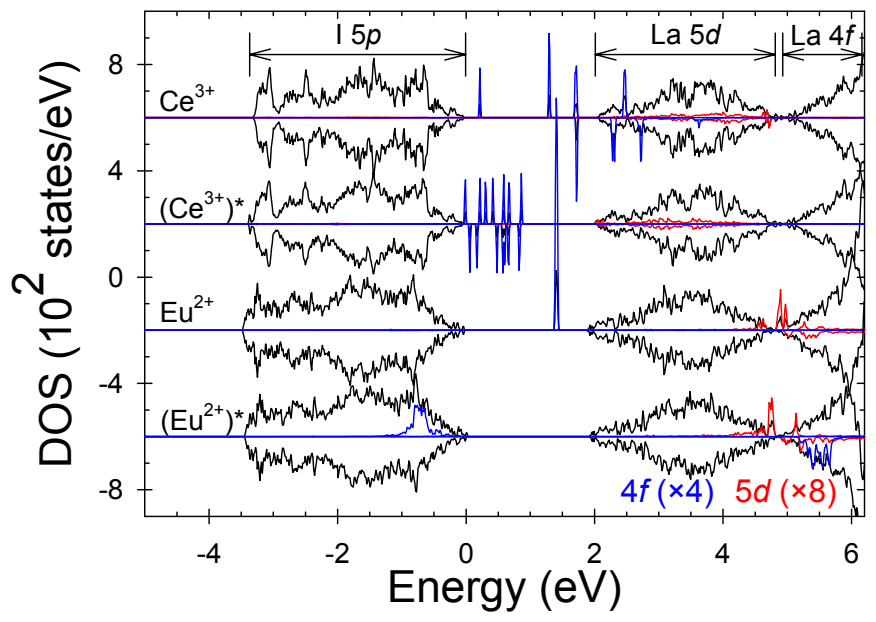

Figure 5: (Color online) Full DOS of $\mathrm{LaI}_{3}$ doped with $\mathrm{Ce}^{3+}$ and $\mathrm{Eu}^{2+}$ in the ground and excited states obtained from GGA $+U$ calculations. Magnified $4 f$ and $5 d$ states of the dopant are shown in blue and red, respectively.

Constrained DFT calculations were performed to approximate the electronic structures of $\left(\mathrm{Ce}^{3+}\right)^{*}$ and $\left(\mathrm{Eu}^{2+}\right)^{*}$ with $4 f^{0} 5 d^{1}$ and $4 f^{6} 5 d^{1}$ configurations, respectively. In both cases, approximately half a dozen bands with $5 d$ character in the ground state were tested. Typical densities of states obtained from these calculations are shown in Fig. 5 together with the corresponding ground-state calculations for comparison. In the ground-state calculations, the $5 d$ states of $\mathrm{Ce}^{3+}$ are significantly delocalized and mix with the $5 d$ states of the La atoms. Therefore, bands near the CBM were selected for the constrained calculations with Ce. In contrast, $\mathrm{Eu}^{2+}$ exhibits more localized $5 d$ states because these states are positioned approximately 5 $\mathrm{eV}$ above the VBM, in between the $5 d$ and $4 f$ states of the La atoms, where the density of states is low and the extent of hybridization is therefore minimal. Consequently, bands with significant Eu $5 d$ character as well as bands near the CBM were selected for the constrained calculations with Eu. For both Ce and Eu, there appears to be little difference between the ground- and excited-state calculations 
in terms of the intensity and position of the $5 d$ states. As expected based on the DOS, Fig. 6 shows that the constrained state is delocalized over the majority of the cations when $\mathrm{LaI}_{3}$ is doped with $\mathrm{Ce}^{3+}$, and the fraction of the partial charge density within the Voronoi volume of $\mathrm{Ce}, \rho_{\mathrm{Ce}}$, is 0.02 . Similar results were obtained for $\mathrm{Eu}$ when selecting bands near the CBM. However, when selecting states with a greater Eu $5 d$ character, a much more localized state was obtained with $\rho_{\text {Eu }}=0.29$. Overall, a more localized excited state can be obtained with $\mathrm{Eu}^{2+}$ compared to the Ce reference case, which would be beneficial to luminescence; but these states lie well above the $\mathrm{CBM}$, as predicted by $\mathrm{GGA}+U$. It is possible they would be found closer to the CBM, if the band gap energy was accurately reproduced.

\section{3. $n s^{2}$ dopants}

\subsubsection{Ground-state electronic structures}

As for the lanthanide dopants, constant-volume geometry optimizations were performed for a $\mathrm{LaI}_{3}$ supercell with one La atom substituted by $\mathrm{Tl}, \mathrm{Pb}, \mathrm{Bi}$, or $\mathrm{Sb}$. To account for the differences in nominal charge between $\mathrm{Tl}^{+}$or $\mathrm{Pb}^{2+}$ and $\mathrm{La}^{3+}$, two and one electrons were added to the calculations, respectively. Information on the structure of the first coordination shells of the dopants is given in Table 3 on their respective pure iodide compounds and, indeed,395 Tl-I nearest-neighbor distances are calculated to be 0.15 $\AA$ longer than La-I distances. $\mathrm{Pb}^{2+}, \mathrm{Bi}^{3+}$, and $\mathrm{Sb}^{3+}$ are all 6-fold coordinated in their pure iodide structures, but Due to the increase in coordination number, longer bonds $\mathrm{s}_{400}$ are formed in the GGA $+U$ calculations and the change in average bond distances relative to those in the corresponding pure iodide compounds is found to be close to linear.

Fig. 7] shows the full DOS obtained from the GGA $+U_{405}$ ground-state calculations of $\mathrm{LaI}_{3}$ doped with these ions,
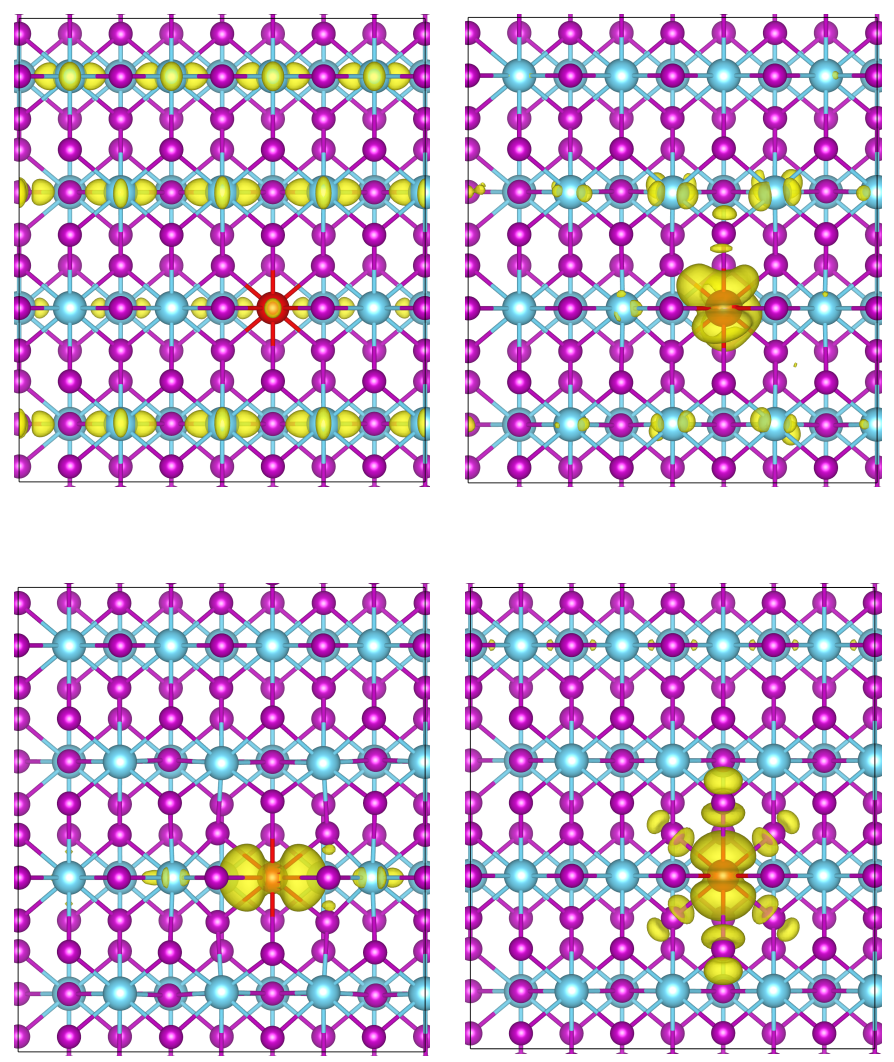

Figure 6: (Color online) Partial charge density of the electron in the constrained (excited) state from the GGA $+U$ calculations of $\left(\mathrm{Ce}^{3+}\right)^{*}$ (top left), $\left(\mathrm{Eu}^{2+}\right)^{*}$ (top right), and $\left(\mathrm{Tl}^{+}\right)^{*}$ (bottom left) and from the HSE calculation of $\left(\mathrm{Bi}^{3+}\right)^{*}$ (bottom right). Iso-surfaces for $0.001 e / \AA^{3}$ are shown in all cases.

with the sum of the $s$ and $p$ states of the dopants magnified. $\mathrm{Pb}^{2+}$ does not exhibit any states in the band gap of the host and, therefore, is not expected to be a suitable activator. In contrast, $\mathrm{Tl}^{+}$has occupied $6 s$ states located just above the VBM and can therefore promote hole capture from the valence band. Both $\mathrm{Sb}^{3+}$ and $\mathrm{Bi}^{3+}$ have unoccupied $p$ states positioned below the CBM and, therefore, could serve as efficient electron traps. Fig. 7 also shows the DOS obtained from static HSE calculations with optimized GGA $+U$ geometries. The fraction of exact exchange obtained from matching the experimental band gap ( $\alpha=0.35)$ was used in these calculations. Unlike in the case of the lanthanide dopants, where increasing the fraction of exact exchange was detrimental to determin- 
Table 3: Coordination numbers $(\mathrm{CN})$ and averages $(r)$ and standard deviations $(\sigma)$ of the M-I nearest-neighbor distances in calculations of doped $\mathrm{LaI}_{3}$ and in corresponding pure $\mathrm{M}$ iodide compounds from experimental crystal structures 37, 35.

\begin{tabular}{cccccccc}
\hline \hline & \multicolumn{3}{c}{${\text { in } \mathrm{LaI}_{3} \text { (calc.) }}$ in $\mathrm{M}^{+\mathrm{x}} \mathrm{I}_{\mathrm{x}}$ (exp.) } \\
\hline $\mathrm{M}$ & $\mathrm{CN}$ & $r(\AA)$ & $\sigma(\AA)$ & $\mathrm{CN}$ & $r(\AA)$ & $\sigma(\AA)$ \\
\hline $\mathrm{La}$ & 8 & 3.35 & 0.11 & 8 & 3.29 & 0.11 \\
$\mathrm{Tl}$ & 8 & 3.50 & 0.12 & 7 & 3.58 & 0.20 \\
$\mathrm{~Pb}$ & 8 & 3.41 & 0.13 & 6 & 3.22 & 0.00 \\
$\mathrm{Bi}$ & 8 & 3.32 & 0.15 & 6 & 3.08 & 0.04 \\
$\mathrm{Sb}$ & 8 & 3.28 & 0.18 & 6 & 3.00 & 0.35 \\
\hline \hline
\end{tabular}

ing the positions of the $4 f$ states accurately, the use of a hybrid exchange-correlation functional does not significantly affect the positions of the $s$ and $p$ states relative to the GGA $+U$ calculations. As a result, the positions of the energy levels obtained with HSE confirm the results of the GGA $+U$ calculations; specifically, despite the increased band gap energy, the unoccupied $p$ states of $\mathrm{Sb}^{3+}$ and $\mathrm{Bi}^{3+}$ are found at approximately the same positions relative to the CBM. Therefore, based on the ground-state ${ }_{430}$ calculations, $\mathrm{Tl}^{+}, \mathrm{Bi}^{3+}$, and $\mathrm{Sb}^{3+}$ are predicted to be potential recombination centers and their excited-state electronic structures will be presented next.

\subsubsection{Excited-state electronic structures}

Constrained DFT calculations were performed for $\mathrm{LaI}_{3}{ }^{45}$ doped with $\mathrm{Tl}^{+}$, where a $6 s$ state was set to be unoccupied and a low-lying conduction band state or a band with $6 p$ character at approximately $5 \mathrm{eV}$ above the VBM in the ground-state calculation were manually filled. Constrained DFT calculations were also carried out for $\mathrm{LaI}_{3}{ }^{440}$ doped with $\mathrm{Bi}^{3+}$ and $\mathrm{Sb}^{3+}$, where a state at the VBM was emptied and a $6 p$ or $5 p$ state of the dopant was set to be occupied, respectively. The DOS obtained from both GGA $+U$ and HSE calculations are shown in Fig. 8. For
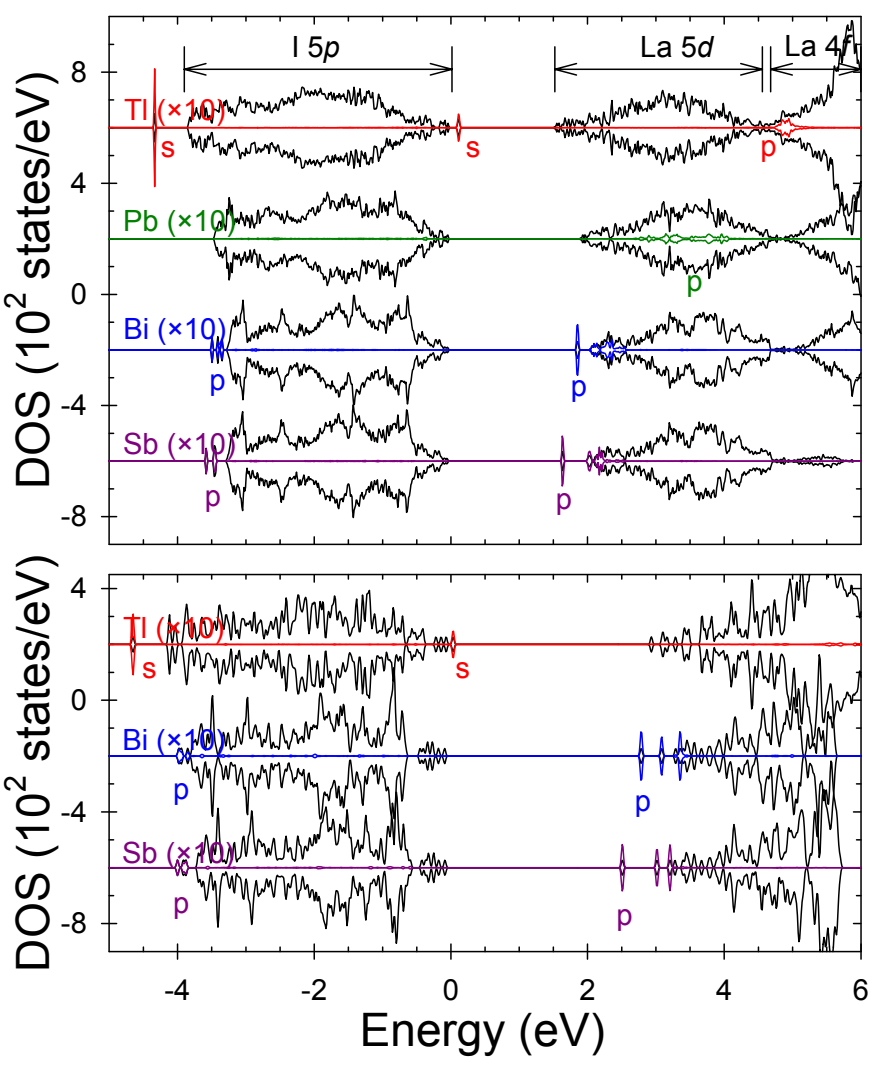

Figure 7: (Color online) Full DOS of $\mathrm{LaI}_{3}$ doped with $\mathrm{n}^{2}$ ions $\left(\mathrm{Tl}^{+}\right.$, $\mathrm{Pb}^{2+}, \mathrm{Bi}^{3+}$, and $\mathrm{Sb}^{3+}$ ) in the ground state from (top) GGA+U and (bottom) HSE $(\alpha=0.35)$ calculations. Magnified $s$ and $p$ states of the dopants are shown in color and labeled.

$\mathrm{Tl}$, the case where a band with $6 p$ character was selected is presented and discussed, as delocalized states were obtained when selecting states near the CBM. In this case, the DOS shows little difference with that obtained from the ground-state calculation (Fig. 7), except for a small splitting of the occupied $s$ states below the valence band; but the electron in the constrained state is well localized on the $\mathrm{Tl}$ dopant $\left(\rho_{\mathrm{Tl}}=0.15\right)$, as shown in Fig. 6. For $\mathrm{Bi}$ and $\mathrm{Sb}$, the occupied $6 p$ or $5 p$ states, respectively, are positioned immediately below the CBM in the GGA+U calculations, with a hole at the VBM. In the HSE calculations, the occupied $p$ states are found at approximately the same position above the VBM; but, because the band gap energy is increased, they are now well below the CBM. Additionally, using the Bi-doped case as calculated with 


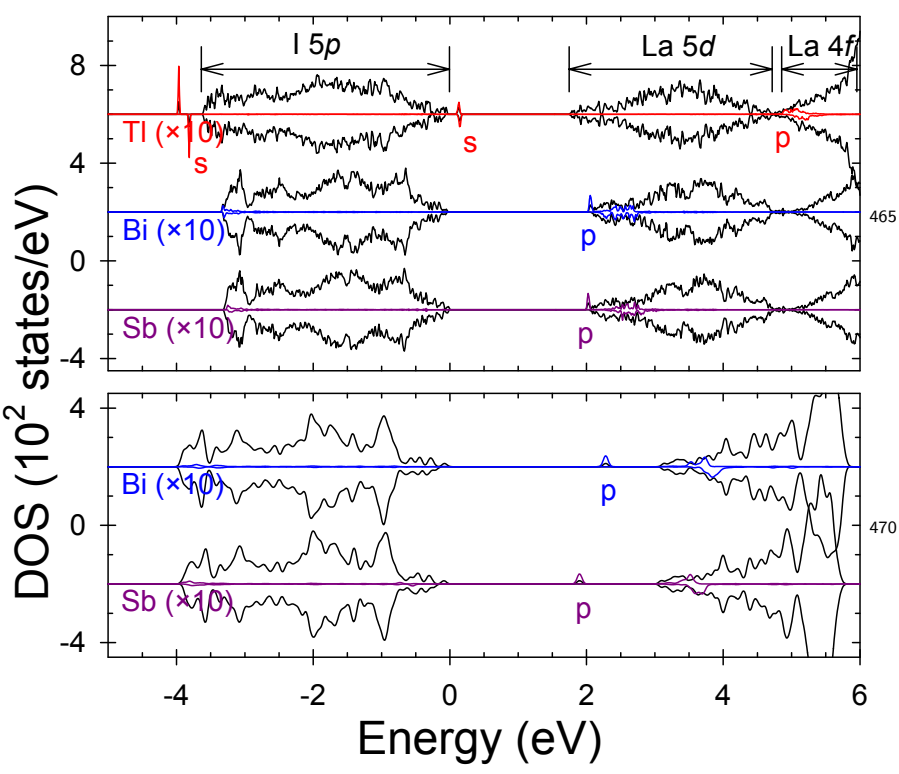

475

Figure 8: (Color online) Full DOS of $\mathrm{LaI}_{3}$ doped with $\mathrm{Tl}^{+}, \mathrm{Bi}^{3+}$, and $\mathrm{Sb}^{3+}$ in the excited state from (top) GGA $+U$ and (bottom) HSE $(\alpha=0.35)$ calculations. Magnified $s$ and $p$ states of the dopants are shown in color and labeled.

HSE as an example, Fig. 6 shows that this gap state is well localized on the dopant $\left(\rho_{\mathrm{Bi}}=0.25\right)$. Overall, $\mathrm{Bi}^{3+}$ and $\mathrm{Sb}^{3+}$ are predicted to be efficient and stable electron traps that can associate with a hole in the valence band to form a localized luminescence center.

\section{Discussion}

A trend in the relative positions of the occupied $4 f$ states of the lanthanide dopants is apparent in Fig. 4. This trend can be directly compared with predictions derived from the empirical relationships established by Dorenbos, as shown in Fig. 9. Specifically, the approach and data reported by Dorenbos [33, 38, are used here to determine the host referred binding energies (HRBE) of the $4 f$ and $5 d$ states of the lanthanide dopants. The $4 f$-VBM gap of 0.26 $\mathrm{eV}$ for Ce was used to set the HRBE of the $4 f^{n}$ state of ${ }_{480}$ of the other $\mathrm{Ln}^{3+}$ ions were obtained using the relative positions reported by Dorenbos 38 and derived from a very wide range of compounds. The HRBE obtained from an earlier version of the empirical relationship [39] is also shown in Fig. 9 for comparison. The position of the $5 d$ state of each $\operatorname{Ln}^{3+}$ was obtained by adding to the HRBE of the corresponding $4 f$ state the energy of the first spinallowed $4 f-5 d$ transition in the free $\mathrm{Ln}^{3+}$ ion, provided by Dorenbos [38, and subtracting the redshift for $\mathrm{Ln}^{3+}$ ions in $\mathrm{LaI}_{3}(3.17 \mathrm{eV})$. The latter was calculated by subtracting the $4 f-5 d$ transition energy of $\mathrm{Ce}^{3+}(2.95 \mathrm{eV})$ obtained in [17] from the excitation spectrum of Ce-doped $\mathrm{LaI}_{3}$ at $6 \mathrm{~K}$ (500 nm excitation was monitored) from that for $\mathrm{Ce}^{3+}$ in the gas phase $(6.12 \mathrm{eV})$. In other words, the redshift was assumed to be the same for all trivalent lanthanide ions 38 .

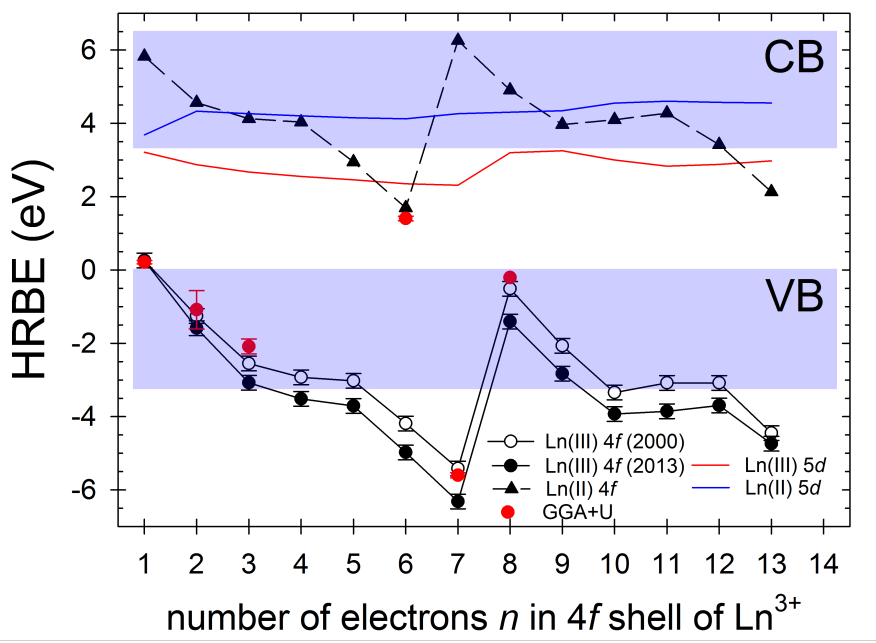

Figure 9: (Color online) Host referred binding energies (HRBE) of the $4 f$ and $5 d$ levels of the lanthanide dopants as a function of the number of electrons in the $4 f$ shell of the trivalent lanthanides as obtained from the GGA $+U$ calculations and Dorenbos' empirical relationships.

The energy of charge transfer to $\mathrm{Eu}^{3+}$ in $\mathrm{LaI}_{3}$ estimated by Dorenbos 33. was used to set the HRBE of the $4 f^{n}$ state of $\mathrm{Ln}^{2+}$ for $n=6$. As was done for the $\mathrm{Ln}^{3+}$ ions, the relative positions reported by Dorenbos [38] were then used to determine the HRBE of the $4 f^{n}$ states of the 
other $\mathrm{Ln}^{2+}$ ions. The same relationship as employed for ${ }_{520}$ the trivalent lanthanide ions was used to determine the positions of the $5 d$ states of the divalent lanthanide ions using the data for free $\mathrm{Ln}^{2+}$ ions [38] and the redshift for $\mathrm{Ln}^{2+}$ ions in $\mathrm{LaI}_{3}(1.79 \mathrm{eV})$, whereby the latter was calculated based on the value of the redshift for $\mathrm{Ln}^{3+}$ using ${ }_{525}$ the empirical correlation between the energy of the $4 f-5 d$ transition of $\mathrm{Ce}^{3+}$ to that of $\mathrm{Eu}^{2+}$ established by Dorenbos 40. This result shows that the $5 d$ states of the divalent lanthanides are found higher above the CBM than those of the trivalent lanthanides, which is consistent with the 530 GGA $+U$ calculations presented in Fig. 5. Overall, good agreement is obtained with the empirical relationships derived by Dorenbos with a single value of $U_{\text {eff }}$ across the lanthanide series. Notably, the HRBE of the $4 f$ state of $\mathrm{Eu}^{2+}$ is in very good agreement with that estimated by 535 Dorenbos 33].

The energy levels presented in Fig. 9 confirm the conclusion drawn from the GGA $+U$ calculations, namely, that none of the trivalent lanthanides are predicted to offer an improvement over $\mathrm{Ce}^{3+}$. The uncertainty of a few tenths540 of an $\mathrm{eV}$ on the position of the $4 f$ level of $\mathrm{Ce}^{3+}$ (Sec. 3.2 .1 is not expected to affect this conclusion as all the other trivalent lanthanide ions have $4 f$ states well below the VBM, except for $\mathrm{Tb}^{3+}$. However, $\mathrm{Tb}^{3+}$ is predicted to have $5 d$ states that are too high in energy to constitute an improvement over $\mathrm{Ce}^{3+}$. Additionally, Fig. 9 confirms that, although it has occupied $4 f$ states well positioned in ${ }^{545}$ the band gap of the host, the potential of $\mathrm{Eu}^{2+}$ to serve as an efficient activator for $\mathrm{LaI}_{3}$ is limited by the fact that its $5 d$ states are above the CBM. However, the ability of $\mathrm{Eu}^{2+}$ to form more localized excited states than $\mathrm{Ce}^{3+}$ in $\mathrm{LaI}_{3}$ could be exploited by increasing the band gap energy through substitution of a portion of the iodide ions by bromide ions. The possibility that an increase of the band gap energy would cause a greater extent of overlap of the $5 d$ states of $\mathrm{Eu}^{2+}$ with the $5 d$ or $4 f$ of the La atoms, and thus a more delocalized excited state, cannot be ruled ${ }^{555}$ out. Mixed $\operatorname{LaX}_{3}(\mathrm{X}=\mathrm{Br}$ or $\mathrm{I})$ compositions were tested experimentally by Birowosuto et al. [41] but using $\mathrm{Ce}^{3+}$ as activator.

Having both the hole and the electron of the excited state located on the dopant and within the band gap of the host is generally thought to be required for dopants to act as efficient activators. However, Du [18] recently proposed an alternative scintillation mechanism based on first-principles calculations of Tl-doped CsI and NaI. In this mechanism, which Du [18] argued is active in Tl-doped CsI, a common and fairly bright scintillator, an electron trapped at a $n s^{2}$ dopant can associate with a hole in the valence band to form a localized excited state that relaxes through a $6 p^{*}$ to $\mathrm{V}_{k}$ emission. This mechanism lifts the requirement to have both the hole and the electron of the excited state located on the dopant and, therefore, could provide a way to exploit low-band gap scintillators such as $\mathrm{LaI}_{3}$, as explicitly noted by $\mathrm{Du}$ [18]. The calculations performed in this work support Du's hypothesis and show that $\mathrm{Bi}^{3+}$ and $\mathrm{Sb}^{3+}$ should lead to the formation of such luminescence centers.

\section{Conclusions}

First-principles calculations were employed to conduct a theory-based search for dopants that would significantly improve the brightness of $\mathrm{LaI}_{3}$, a low-band gap scintillator with a potentially very high light output not yet exploited. Two families of dopants were considered: lanthanide ions and $\mathrm{n} s^{2}$ ions. The dopants fell into three categories, based on their predicted effect on the light output relative to $\mathrm{Ce}^{3+}$, for which experimental data exist. The first category included all the trivalent lanthanide dopants other than $\mathrm{Ce}^{3+}$ as well as $\mathrm{Pb}^{2+}$. These dopants are predicted not to yield improvement in brightness due to their lack of both occupied and unoccupied states within the band gap of the host. The second category consisted of $\mathrm{Eu}^{2+}$ and $\mathrm{Tl}^{+}$, which exhibited occupied states above the VBM; but 
also unoccupied states above the CBM. Although the simulated excited states were shown to be significantly localized on the dopant in both cases, the fact that those states ${ }^{595}$ are above the CBM is expected to rule out dopants in the second category as efficient activators. However, because of their proximity to the CBM, dopants in the second category may be efficient activators in cases where the band ${ }^{600}$ gap is slightly increased through the substitution of iodide ions by bromide ions to form $\mathrm{LaI}_{3-x} \mathrm{Br}_{x}$. Finally, $\mathrm{Bi}^{3+}$ and $\mathrm{Sb}^{3+}$ formed the third category, which shows unoccupied states in the band gap; but can associate with a hole in the valence band to form well-localized excited states. Dopants in the third category are predicted to be efficient luminescence centers. Therefore, we recommend Bi- and Sb-doped $\mathrm{LaI}_{3}$ should be grown and tested experimentally.

\section{Acknowledgments}

The authors acknowledge Drs. Luke W. Campbell and615 Yulong Xie for useful discussions. This research was supported by the National Nuclear Security Administration, Office of Nuclear Nonproliferation Research and Engineering (NA-22), of the U.S. Department of Energy (DOE). ${ }^{620}$ The calculations were carried out using PNNL Institutional Computing at Pacific Northwest National Laboratory.

\section{References}

\[1] A. Peurrung, Materials Today 11 (2008) 50-54. doi 10.1016/ S1369-7021(08)70019-1.

[2] W. Setyawan, R. M. Gaume, R. Feigelson, S. Curtarolo, IEEE630 Trans. Nucl. Sci. 56 (2009) 2989-2996. doi 10.1109/TNS. 2009. 2027019

[3] R. T. Williams, J. Q. Grim, Q. Li, K. B. Ucer, W. W. Moses, Phys. Status Solidi B 248 (2011) 426-438. doi 10.1002/pssb. 201000610

[4] P. Erhart, A. Schleife, B. Sadigh, D. Åberg, Phys. Rev. B 89 (2014) 075132. doi 10.1103/PhysRevB.89.075132

[5] A. Vasil'ev, A. Gektin, IEEE Trans. Nucl. Sci. 61 (2014) 235245. doi $10.1109 /$ TNS. 2013.2282117
[6] M. P. Prange, L. W. Campbell, D. Wu, F. Gao, S. Kerisit, Phys. Rev. B 91 (2015) 104305. doi 10.1103/PhysRevB.91.104305

[7] X. Lu, Q. Li, G. A. Bizarri, K. Yang, M. R. Mayhugh, P. R.

Menge, R. T. Williams, Phys. Rev. B 92 (2015) 115207. doi 10. 1103/PhysRevB.92.115207.

[8] M. P. Prange, R. M. Van Ginhoven, N. Govind, F. Gao, Phys. Rev. B 87 (2013) 115101. doi 10.1103/PhysRevB.87.115101

[9] D. Åberg, B. Sadigh, P. Erhart, Phys. Rev. B 85 (2012) 125134. doi $10.1103 /$ PhysRevB.85.125134.

[10] Q. Li, R. T. Williams, D. Åberg, Phys. Status Solidi B 250 (2013) 233-243. doi 10.1002/pssb.201200503

[11] A. Canning, R. Boutchko, A. Chaudhry, S. Derenzo, IEEE 1 Trans. Nucl. Sci. 56 (2009) 944-948. doi 10.1109/TNS.2009. 2019645

[12] A. Canning, A. Chaudhry, R. Boutchko, N. Grønbech-Jensen, Phys. Rev. B 83 (2011) 125115. doi 10.1103/PhysRevB.83. 125115

[13] A. Chaudhry, R. Boutchko, S. Chourou, G. Zhang, N. Grønbech-Jensen, A. Canning, Phys. Rev. B 89 (2014) 155105. doi 10.1103/PhysRevB.89.155105

[14] D. Åberg, B. Sadigh, , A. Schleife, P. Erhart, Appl. Phys. Lett. 104 (2014) 211908. doi 10.1063/1.4880576

[15] R. Adhikari, Q. Li, R. T. Williams, A. Burger, K. Biswas, J. Appl. Phys. 116 (2014) 223703. doi 10.1063/1.4903766

[16] P. Erhart, B. Sadigh, A. Schleife, D. Åberg, Phys. Rev. B 91 (2015) 165206. doi 10.1103/PhysRevB.91.165206

[17] A. Bessiere, P. Dorenbos, C. van Eijk, K. Krämer, H. Güdel, C. de Mello Donega, A. Meijerink, Nucl. Instrum. Meth. Phys. Res. A 537 (2005) 22-26. doi 10.1016/j.nima.2004.07.224

[18] M. H. Du, J. Mater. Chem. C 2 (2014) 4784-4791. doi:10.1039/ C4TC00485J

[19] G. Kresse, J. Hafner, Phys. Rev. B 47 (1993) 558-561. doi 10. 1103/PhysRevB.47.558

[20] G. Kresse, J. Furthmüller, Phys. Rev. B 54 (1996) 11169-11186. doi 10.1103/PhysRevB.54.11169

[21] G. Kresse, J. Furthmüller, Comput. Mater. Sci. 6 (1996) 15-50. doi 10.1016/0927-0256(96)00008-0.

[1.22] P. E. Blöchl, Phys. Rev. B 50 (1994) 17953-17979. doi:10.1103/ PhysRevB.50.17953

[23] G. Kresse, D. Joubert, Phys. Rev. B 59 (1999) 1758-1775. doi $10.1103 /$ PhysRevB.59.1758

[24] J. P. Perdew, K. Burke, M. Ernzerhof, Phys. Rev. Lett. 77 (1996) 3865-3868. doi 10.1103/PhysRevLett.77.3865

[25] S. L. Dudarev, G. A. Botton, S. Y. Savrasov, C. J. Humphreys, A. P. Sutton, Phys. Rev. B 57 (1998) 1505-1509. doi 10.1103/ PhysRevB.57.1505

[26] A. Rohrbach, J. Hafner, G. Kresse, J. Phys.: Condens. Matter 15 (2003) 979. doi $10.1088 / 0953-8984 / 15 / 6 / 325$ 
[27] M. T. Czyzyk, G. A. Sawatzky, Phys. Rev. B 49 (1994) 1421114228. doi 10.1103/PhysRevB.49.14211

[28] A. V. Krukau, O. A. Vydrov, A. F. Izmaylov, G. E. Scuseria, J. Chem. Phys 125 (2006) 224106. doi 10.1063/1.2404663.

[29] M. Shishkin, M. Marsman, G. Kresse, Phys. Rev. Lett. 99 (2007) 246403. doi 10.1103/PhysRevLett.99.246403

[30] M. Shishkin, G. Kresse, Phys. Rev. B 75 (2007) 235102. doi 10. 1103/PhysRevB.75.235102

[31] L. B. Asprey, T. K. Keenan, F. H. Kruse, Inorg. Chem. 3 (1964) 1137-1141. doi 10.1021/ic50018a015

[32] P. Dorenbos, J. Lumin. 111 (2005) 89-104. doi 10.1016/j. jlumin.2004.07.003

[33] P. Dorenbos, J. Lumin. 136 (2013) 122-129. doi 10.1016/j. jlumin.2012.11.030

655 [34] J. H. Levy, J. C. Taylor, P. W. Wilson, Acta Cryst. B 31 (1975) 880-882. doi $10.1107 /$ S0567740875003986

[35] J. Trotter, T. Zobel, Z. Kristall. 123 (1966) 67-72. doi 10.1524/ Zkri.1966.123.16.67

[36] H. Bärnighausen, N. Schultz, Acta Cryst. 25 (1969) 1104-1110.

660 doi $10.1107 /$ S0567740869003591

[37] R. Wyckoff, Crystal Structures, 2nd ed., Wiley, 1963.

[38] P. Dorenbos, ECS J. Solid State Sci. Technol. 2 (2013) R3001R3011. doi 10.1149/2.001302jss

[39] P. Dorenbos, J. Lumin. 91 (2000) 91-106. doi 10.1016/ S0022-2313(00)00197-6.

[40] P. Dorenbos, J. Phys.: Condens. Matter 15 (2003) 4797-4807. doi $10.1088 / 0953-8984 / 15 / 27 / 311$

[41] M. D. Birowosuto, P. Dorenbos, K. W. Krämer, H. U. Güdel, J. Appl. Phys. 103 (2008) 103517. doi 10.1063/1.2930884 